\title{
Expression of mitochondrial transcription factor $A$ in endometrial carcinomas: clinicopathologic correlations and prognostic significance
}

\author{
Naoyuki Toki • Seiji Kagami • Tomoko Kurita • Toshinori Kawagoe • \\ Yusuke Matsuura • Toru Hachisuga • Atsuji Matsuyama • Hiroshi Hashimoto • \\ Hiroto Izumi • Kimitoshi Kohno
}

Received: 13 October 2009 /Revised: 20 January 2010 /Accepted: 16 February 2010 / Published online: 16 March 2010

(C) The Author(s) 2010. This article is published with open access at Springerlink.com

\begin{abstract}
Mitochondrial transcription factor A (mtTFA) is necessary for both transcription and maintenance of mitochondrial DNA. This study was conducted to elucidate the clinicopathologic and prognostic significance of mtTFA in patients with endometrial carcinoma. This study investigated the relationship between the immunohistochemical expression of mtTFA and various clinicopathological variables in 276 endometrial carcinomas, including 245 endometrioid adenocarcinomas and 31 nonendometrioid carcinomas (21 serous carcinomas and 10 clear cell adenocarcinomas). Both uni- and multivariate regression analyses were performed. The mtTFA labeling index of endometrioid adenocarcinomas ranged from $0 \%$ to $98 \%$, with a median value of $32 \%$, which was selected as the cutoff point for mtTFA expression. The mtTFA expression in endometrioid adenocarcinomas was significantly associated with the surgical stage, myometrial invasion, lymphovas-
\end{abstract}

\footnotetext{
N. Toki $(\bowtie) \cdot$ S. Kagami $\cdot$ T. Kurita $\cdot$ T. Kawagoe $\cdot$ Y. Matsuura

T. Hachisuga

Department of Obstetrics and Gynecology, University of

Occupational and Environmental Health School of Medicine,

1-1, Iseigaoka, Yahatanishi-ku,

Kitakyushu 807-8555, Japan

e-mail: n-toki@med.uoeh-u.ac.jp

A. Matsuyama $\cdot$ H. Hashimoto

Department of Pathology I, University of Occupational and Environmental Health School of Medicine,

1-1, Iseigaoka, Yahatanishi-ku,

Kitakyushu 807-8555, Japan

H. Izumi $\cdot$ K. Kohno

Department of Molecular Biology, University of Occupational and Environmental Health School of Medicine,

1-1, Iseigaoka, Yahatanishi-ku,

Kitakyushu 807-8555, Japan
}

cular space invasion, cervical invasion, and lymph node metastasis. In contrast, no correlation between clinicopathologic variables and mtTFA expression was found in nonendometrioid carcinomas. Correlation analysis between mtTFA and p53 expression by using the Pearson test showed significant correlation in endometrioid adenocarcinomas $(P=0.007)$, but no significant correlation in nonendometrioid carcinomas $(P=0.947)$. A univariate survival analysis showed that the 10 -year overall survival rate of the patients with mtTFA-positive endometrioid adenocarcinoma was significantly worse than that of patients with mtTFA-negative endometrioid adenocarcinoma $(80.8 \%$ vs. $93.8 \%, P=0.012)$. However, the multivariate analysis revealed that mtTFA expression in endometrioid adenocarcinomas was no independent prognostic factor. The positive mtTFA expression is a useful maker for progression of the tumors and the poor prognosis of the patients in endometrioid adenocarcinomas.

Keywords Endometrial cancer - Mitochondria . Mitochondrial transcription factor A $\cdot \mathrm{p} 53$.

Immunohistochemistry

\section{Introduction}

Endometrial carcinoma is the most common malignant tumor of the female genital tract in the Western countries. A dualistic model of endometrial carcinogenesis has been proposed since the $1980 \mathrm{~s}$, based on light microscopic appearance, clinical behavior, and epidemiology [1]. Type I cancers, those with endometrioid histology, comprise approximately $80 \%$ of all endometrial carcinomas. In contrast, type II endometrial cancers have nonendometrioid 
histology (usually serous or clear cell) with aggressive clinical course. Hormonal risk factors have not been identified. Common genetic changes in type I cancers include microsatellite instability or specific mutation of PTEN, K-ras, and $\beta$-catenin genes, while most type II cancers contain mutations of $\mathrm{p} 53$ [2].

Mammalian mitochondrial transcription factor A (mtTFA) proteins have a molecular weight of $\sim 25 \mathrm{kDa}$ and consist of an amino-terminal high mobility group (HMG) domain, a basic linker region, a second HMG domain, and a basic carboxyterminal tail [3]. It was recently demonstrated that mtTFA, which was firstly identified as a mitochondrial transcription factor, is involved in not only the transcription of mitochondrial DNA (mtDNA) but also the replication of mtDNA, recognition of mtDNA damage, stabilization of mtDNA, and indirectly, in repair of mtDNA [4].

Several studies showed that expression of mtTFA increased the amount of mtDNA in association with the total amount of mtTFA in human cells $[3,5]$. Maintenance of mtDNA copy number and expression are considered to be essential for preservation of mitochondrial function and cell growth via the regulation of mtTFA $[3,6]$. A quantitative study using laser-captured microdissected tissues showed an increase of mtDNA content in endometrioid adenocarcinomas [7]. Italian authors reported that 2-fold increase of the mtTFA content was found in the endometrioid adenocarcinoma samples compared to the proliferative control endometrial samples [8]. The down-regulation of mtTFA was also reported to be significantly associated with the disease progress of colorectal cancer [9].

This study investigated the correlation between the expression of mtTFA and the clinicopathological profile of the patients with endometrial carcinoma and thus found the mtTFA expression to be a useful maker for progression of the tumors and the poor prognosis of the patients in endometrioid adenocarcinomas.

\section{Materials and methods}

\section{Case selection}

The study included 276 Japanese patients with endometrial carcinoma who had undergone surgery at University of Occupational and Environmental Health hospital from 1990 to 2006. These included 245 cases of endometrioid adenocarcinoma and 31 cases of nonendometrioid carcinoma (21 cases of serous carcinoma and 10 cases of clear cell adenocarcinoma). All studied endometrial carcinoma specimens were from hysterectomy specimens. Tumor grade and stage were evaluated using FIGO criteria. The retroperitoneal lymphadenectomy or sampling was performed in 211 patients with endometrioid adenocarcinoma and 20 patients with nonendometrioid carcinoma.

Twenty-five control samples were obtained from 25 patients who underwent hysterectomy at University of Occupational and Environmental Health hospital in 2008 for benign uterine lesions, including leiomyomas, adenomyosis, and uterine prolapse.

\section{Immunohistochemistry}

For immunohistchemistry, $4-\mu \mathrm{m}$ sections were cut from formalin-fixed paraffin-embedded tissue blocks, deparaffinized in xylene, and rehydrated through sequential changes of alcohol and distilled water. A polyclonal antibody against mtTFA was generated by multiple immunization of a New Zealand white rabbit using synthetic peptides, as described previously (dilution 1:400) [10]. p53 was detected using the ready-to-use p53 monoclonal antibody (clone DO-7, DAKO, Kyoto, Japan). The slides for p53 were heated in an autoclave at $120^{\circ} \mathrm{C}$ for $5 \mathrm{~min}$ in $0.01 \mathrm{M}$ citrate buffer $(\mathrm{pH}=6.0)$ before the immunostaining. The immunoreactivity for mtTFA was not changed by the autoclave heating system. The slides for mtTFA and p53 were incubated with these antibodies for $2 \mathrm{~h}$ at room temperature. The selected slides were incubated with mtTFA antibody or mtTFA antibody+synthetic peptides of mtTFA $(40 \mu \mathrm{g})$ in order to check the specificity of mtTFA immunostaining. Antibody binding was visualized using the EnVision+ Dual link system and diaminobenzidine as chromogen (Dako Cytomation, Kyoto, Japan). The slides were counterstained with methyl green and mounted.

\section{Interpretation of immunohistochemical preparations}

Immunostained slides were analyzed independently by two authors. Slight differences were resolved by simultaneous viewing. The labeling indices (LI) of mtTFA and p53 were defined as the percentage of the tumor cells with clear cytoplasmic and nuclear immunoreactivity out of the total number of the tumor cells, respectively. LI was determined by counting at least 500 cells in the most active area of the specimen. The cases for mtTFA and p53 were judged as positive when more than $32 \%$ (median) and $50 \%$ of the tumor cells [11] showed immunoreactivity, respectively.

\section{Statistical analysis}

Statistical analyses were carried out using SPSS for Windows, version 16.0.0 (SPSS, Chicago, IL, USA). The Mann-Whitney $U$ test was used for comparison of clinicopathological variables with positive and negative mtTFA expression. The degree of correlation between the p53 and mtTFA expression was evaluated using the Pearson 


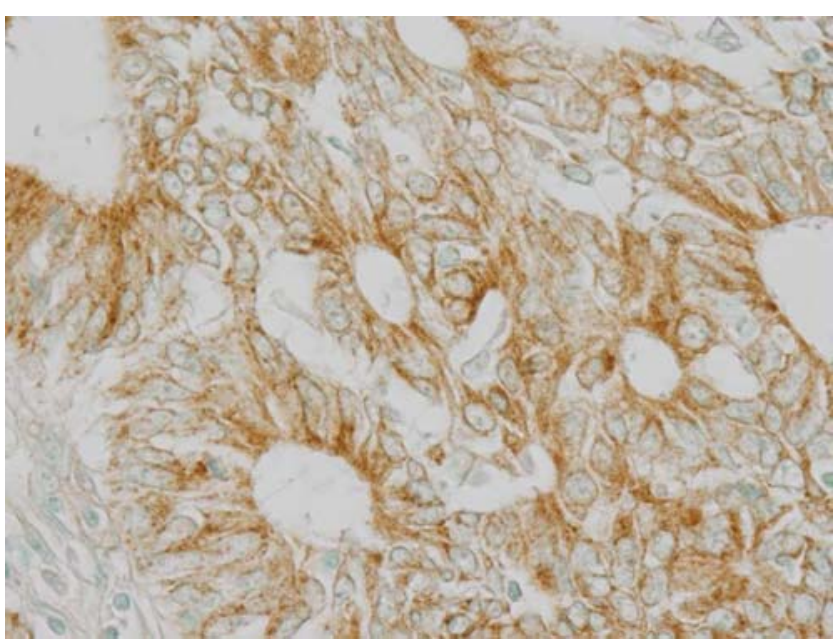

Fig. 1 Immunohistochemical positive mtTFA expression in grade 2 endometrioid adenocarcinoma. $\times 400$

test. The survival time was calculated from date of the initial surgery. The cumulative 10 -year survival was determined using the Kaplan-Meier product-limited method. The log-rank test was used to test the differences in survival within variables. The Cox proportional hazards model was used to identify and simultaneously evaluate any independent prognostic factors associated with relative survival. Statistical significance was considered to exist at a value of $P<0.05$.

\section{Results}

\section{Clinicopathologic features}

Two hundred and forty-five patients with endometrioid adenocarcinoma ranged in age from 27 to 82 years. The mean age was 56.5 years. One hundred and nineteen cases (48.6\%) had tumor grade 1, 82 cases (33.4\%) grade 2 and 44 cases $(18.0 \%)$ grade 3 . FIGO stages of these 245 endometrioid adenocarcinomas were 59 patients $(24.1 \%)$ with stage Ia disease, 87 patients $(35.5 \%)$ with stage $\mathrm{Ib}$ disease, 23 patients $(9.4 \%)$ with stage Ic disease, seven patients $(2.9 \%)$ with stage IIa disease, 11 patients $(4.5 \%)$ with stage IIb disease, 25 patients $(10.2 \%)$ with stage IIIa disease, three patients $(1.2 \%)$ with stage IIIb disease, 26 patients $(10.6 \%)$ with stage IIIc disease, and four patients (1.6\%) with stage IV disease.

The age of the 31 patients with nonendometrioid adenocarcinoma ranged from 53 to 81 years with a mean age of 67.1 years. Among these 31 cases of nonendometrioid adenocarcinoma with clinicopathologic stage assessed, six cases (19.3\%) were diagnosed with stage Ia disease, five (16.1\%) stage $\mathrm{Ib}$ disease, three $(9.7 \%)$ stage Ic disease, three $(9.7 \%)$ stage IIa disease, three $(9.7 \%) \mathrm{IIb}$ disease, four $(12.9 \%)$ stage IIIa disease, one (3.2\%) stage IIIb disease, three $(9.7 \%)$ stage IIIc disease, and three $(9.7 \%)$ stage IV disease.

The age of the 25 control patients ranged from 32 to 72 years. Of these 25 cases, 10 had proliferative, 10 secretory, and five atrophic endometria.

Immunohistochemical findings

\section{mtTFA expression}

mtTFA showed only cytoplasmic expression (Fig. 1). The specificity of this mtTFA polyclonal antibody was tested by immunohistochemisry. After incubation of this antibody with the excess of synthesized peptides of mtTFA, the positive immunostaining was almost abolished (Fig. 2). Of 245 endometrioid adenocarcinomas, the mean mtTFA index
Fig. 2 Immunohistochemical mtTFA expression in grade 1 endometrioid adenocarcinoma after incubation of an antimtTFA polyclonal antibody (a) or an anti-mtTFA polyclonal antibody + synthesized peptides of mtTFA (b). $\times 200$
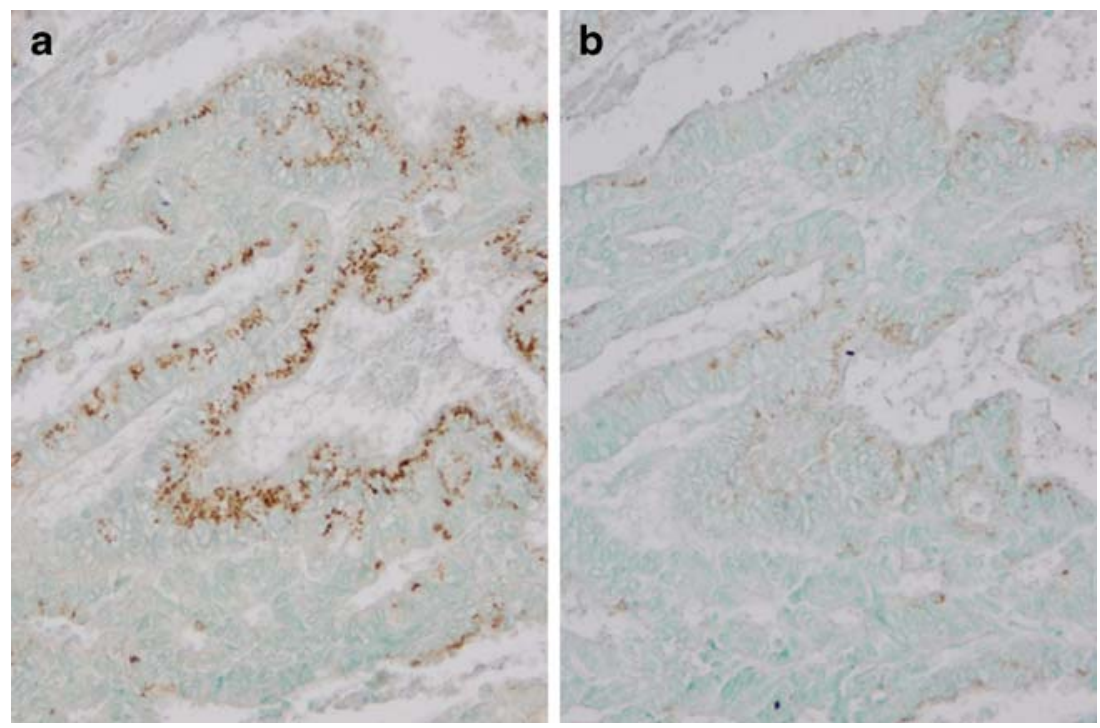
was $21 \%$, and the median was $32 \%$. The positive mtTFA expression ( $\mathrm{LI} \geq 32 \%)$ was found in $52(43.7 \%)$ of 119 grade 1 tumors, $43(52.4 \%)$ of 82 grade 2 tumors, and 28 (63.6\%) of 44 grade 3 tumors. The mtTFA expression correlated significantly with the surgical stage, myometrial invasion, lymphovascular space invasion (LVSI), cervical invasion, lymph node metastasis, and p53 expression but did not correlate with age of the patients, grade of the tumor, and ovarian metastasis (Table 1).

The positive cytoplasmic staining for mtTFA was shown in $17(54.8 \%)$ of 31 nonendometrioid carcinomas (11 of 21 serous carcinomas and six of 10 clear cell adenocarcinomas). There was no significant correlation between mtTFA expression and any clinicopathologic variables in nonendometrioid carcinomas.

Of the 25 control cases, the cytoplasmic staining for mtTFA was positive for the vascular endothelial cells and was negative in the stromal cells and the atrophic endometrial glands. The proliferative and secretory endometrial glands and the smooth muscle cells were weakly positive for mtTFA.

\section{p53 expression}

Positive immunohistochemical reaction for p53 in tumor cells was characterized by positive staining seen in the

Table 1 Clinicopathologic variables and mtTFA expression

\begin{tabular}{|c|c|c|c|c|c|c|}
\hline \multirow[t]{3}{*}{ Variable } & \multicolumn{3}{|c|}{ mtTFA in } & \multicolumn{3}{|c|}{ mtTFA in } \\
\hline & \multicolumn{3}{|c|}{ Endometrioid adenocarcinomas } & \multicolumn{3}{|c|}{ Nonendometroioid carcinomas } \\
\hline & $\begin{array}{l}\text { Positive } \\
n=123\end{array}$ & $\begin{array}{l}\text { Negative } \\
n=122\end{array}$ & $P$ value & $\begin{array}{l}\text { Positive } \\
n=17\end{array}$ & $\begin{array}{l}\text { Negative } \\
n=14\end{array}$ & $P$ value \\
\hline Age & & & 0.376 & & & 0.608 \\
\hline$<60$ years & 76 & 82 & & 4 & 2 & \\
\hline$\geq 60$ years & 47 & 40 & & 13 & 12 & \\
\hline Stage & & & 0.008 & & & 0.197 \\
\hline I and II & 85 & 102 & & 13 & 7 & \\
\hline III and IV & 38 & 20 & & 4 & 7 & \\
\hline Grade & & & 0.050 & & & \\
\hline 1 and 2 & 95 & 106 & & & & \\
\hline 3 & 28 & 16 & & & & \\
\hline Myometrial invasion & & & $<0.001$ & & & 0.175 \\
\hline$\geq 1 / 2$ & 78 & 103 & & 11 & 5 & \\
\hline$>1 / 2$ & 45 & 19 & & 6 & 9 & \\
\hline LVSI & & & 0.006 & & & 0.953 \\
\hline- & 68 & 88 & & 7 & 6 & \\
\hline+ & 55 & 34 & & 10 & 8 & \\
\hline Cervical invasion & & & 0.015 & & & 0.983 \\
\hline- & 97 & 110 & & 12 & 10 & \\
\hline+ & 26 & 12 & & 5 & 4 & \\
\hline Ovarian metastasis & & & 0.542 & & & 0.856 \\
\hline- & 109 & 111 & & 14 & 12 & \\
\hline+ & 14 & 11 & & 3 & 2 & \\
\hline Lymph node metastasis ${ }^{\mathrm{a}}$ & & & 0.003 & & & 0.672 \\
\hline- & 87 & 100 & & 8 & 7 & \\
\hline+ & 19 & 5 & & 2 & 3 & \\
\hline LI ofp53 expression & & & 0.003 & & & 0.73 \\
\hline$<50 \%$ & 98 & 113 & & 2 & 1 & \\
\hline$\geq 50 \%$ & 25 & 9 & & 15 & 13 & \\
\hline
\end{tabular}

mtTFA mitochondrial transcription A, LVSI lymphovascular space invasion, LI labeling index

${ }^{a}$ The retroperitoneal lymph node adenectomy or sampling was done in 211 endometrioid cases and 20 nonendometrioid cases 
nucleus (Fig. 3). Among 245 endometrioid adenocarcinomas, the range of LI for p53 expression was from $0 \%$ to $98 \%$. Seventy-seven tumors $(31.4 \%)$ demonstrated in $0 \%$ of LI, $41(16.7 \%)$ tumors in $1-9 \%$ of LI, $92(37.6 \%)$ tumors in $10-49 \%$ of LI, and $35(14.3 \%)$ tumors in $\geq 50 \%$ of LI. Among 31 nonendometrioid carcinomas, the range of LI for p53 expression was from $20 \%$ to $98 \%$. Three tumors (9.7\%) demonstrated in $20-49 \%$ of LI and 28 (90.3\%) tumors in $\geq 50 \%$ of LI. Correlation analysis between mtTFA and p53 expression by using the Pearson test showed significant correlation in endometrioid adenocarcinomas $(P=0.007)$, but no significant correlation in nonendometrioid carcinomas $(P=0.947)$. All 25 samples of morphologically benign endometrium evaluated were negative for $\mathrm{p} 53$.

\section{Prognosis}

Figure 4 shows the Kaplan-Meier survival curve for 211 patients with endometrioid adenocarcinoma, who were performed the retroperitoneal lymphadectomy or sampling. The 10-year overall survival rate of patients with mtTFAnegative endometrioid adenocarcinoma was $93.8 \%$. In contrast, the 10-year survival rate of patients with mtTFApositive endometrioid adenocarcinoma was $80.8 \%$. Patients with mtTFA-positive endometrioid tumor showed a lower long-term survival rate in comparison to patients with mtTFA-negative endometrioid tumor $(P=0.012, \log$-rank test). The 10-year overall survival rates of patients with p53-negative $(\mathrm{LI}<50 \%)$ and $\mathrm{p} 53$-positive ( $\mathrm{LI} \geq 50 \%)$ endometrioid adenocarcinomas was $89.9 \%$ and $72.2 \%(P<0.03$, log-rank test). A univariate analysis showed that age of the patients, the grade of the tumor, myometrial invasion, LVSI, cervical invasion, ovarian metastasis, lymph node metastasis, p53 expression, and mtTFA expression were

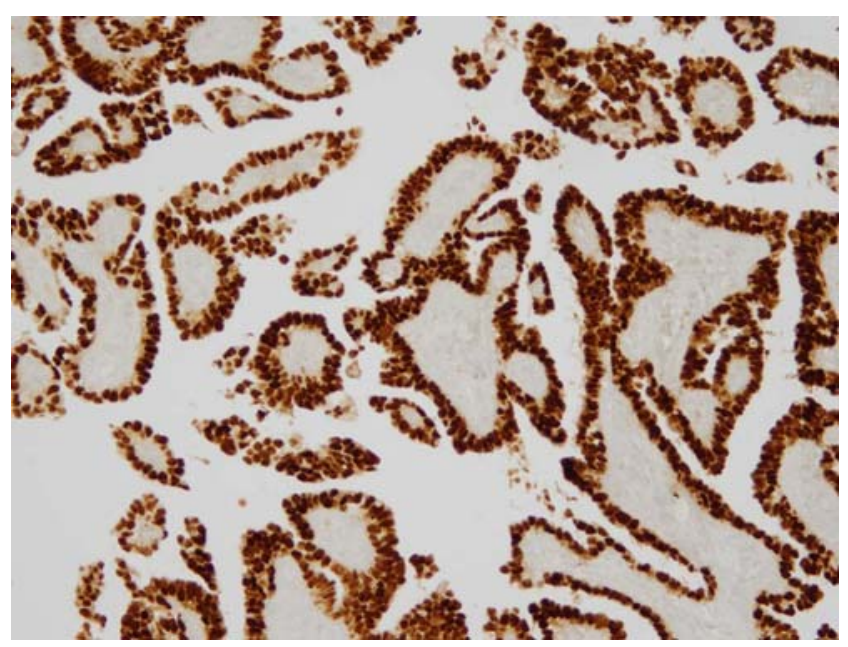

Fig. 3 Immunohistochemical positive p53 expression in serous carcinoma. $\times 100$

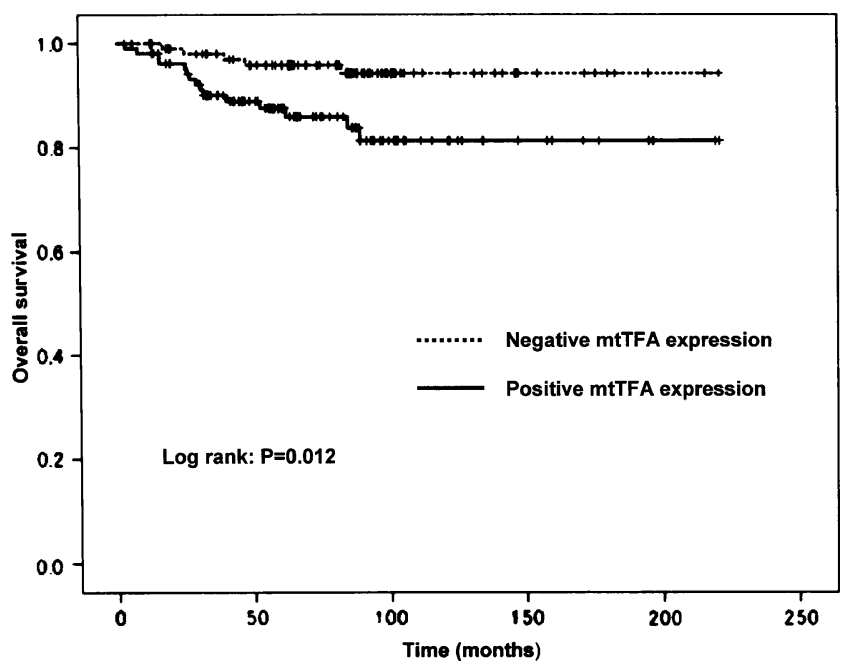

Fig. 4 Comparison of the overall survival rates in 211 patients with endometrioid adenocarcinoma according to mtTFA expression

significantly associated with survival (Table 2). In a multivariate analysis using the Cox proportional hazards model, the significance of myometrial invasion and lymph node metastasis was preserved, whereas the significance of the age of the patients, the grade of the tumor, LVSI, cervical invasion, ovarian metastasis, p53 expression, and mtTFA expression disappeared.

Of 31 patients with nonendometrioid carcinoma, the 10 -year overall survival rates of patients with mtTFAnegative tumor and with mtTFA-positive tumor were $42.3 \%$ and $61.2 \%$, respectively $(P=0.40$, log-rank test). In a univariate analysis, the significance of surgical stage, LVSI, and ovarian metastasis was demonstrated $(P=0.001,0.014$, and 0.006 , respectively), whereas the significance of age of the patients, myometrial invasion, cervical invasion, lymph node metastasis, p53 expression, and mtTFA expression was not demonstrated.

\section{Discussion}

In the present study, a univariate survival analysis showed that the survival rate of patients with mtTFA-negative endometrioid adenocarcinoma was significantly better than that for patients with mtTFA-positive endometrioid adenocarcinoma. The mtTFA expression was significantly associated with unfavorable histopathological variables of endometrioid adenocarcinomas including an advanced stage. The increases of mtDNA content [7] or mtTFA [8] were reported in endometrioid adenocarcinomas. The mtDNA copy number is suggested to be increased by a feedback mechanism that compensates for defects in mitochondria harboring mutated mtDNA and a defective respiratory system [12]. The inhibition of mtTFA activity 
Table 2 Uni- and multivariate analyses of prognostic variables for overall survival of the patients with endometrioid adenocarcinoma by Cox proportional hazards model
LVSI lymphovascular space invasion, mtTFA mitochodrial transcription factor A, $H R$ hazard ratio, $L I$ labeling index, 95\% CI $95 \%$ confidence interval

\begin{tabular}{|c|c|c|c|c|c|c|}
\hline \multirow[t]{2}{*}{ Variables } & \multicolumn{3}{|c|}{ Univariate analysis } & \multicolumn{3}{|c|}{ Multivariate analysis } \\
\hline & HR & $95 \% \mathrm{CI}$ & $P$ value & HR & $95 \% \mathrm{CI}$ & $P$ value \\
\hline \multicolumn{7}{|l|}{ Age } \\
\hline$<60$ vs. $\geq 60$ years & 2.742 & $1.134-6.630$ & 0.025 & 1.527 & $0.602-3.874$ & 0.373 \\
\hline \multicolumn{7}{|l|}{ Grade } \\
\hline 1 and 2 vs. 3 & 3.476 & $1.419-8.513$ & 0.006 & 1.235 & $0.339-4.505$ & 0.749 \\
\hline \multicolumn{7}{|l|}{ Myometrial invasion } \\
\hline$\geq 1 / 2$ vs. $>1 / 2$ & 17.997 & $5.272-61.438$ & $<0.001$ & 6.545 & $1.517-28.246$ & 0.012 \\
\hline \multicolumn{7}{|l|}{ LVSI } \\
\hline Negative vs. positive & 5.876 & $2.133-16.183$ & $<0.001$ & 0.969 & $0.232-4.037$ & 0.965 \\
\hline \multicolumn{7}{|l|}{ Cervical invasion } \\
\hline Negative vs. positive & 4.560 & $1.815-11.458$ & 0.001 & 1.085 & $0.306-3.843$ & 0.899 \\
\hline \multicolumn{7}{|l|}{ Ovarian metastasis } \\
\hline Negative vs. positive & 5.955 & $2.373-14.941$ & $<0.001$ & 3.065 & $0.988-9.504$ & 0.052 \\
\hline \multicolumn{7}{|l|}{ Lymph node metastasis } \\
\hline Negative vs. positive & 15.738 & $6.401-38.693$ & $<0.001$ & 4.954 & $1.510-16.256$ & 0.008 \\
\hline \multicolumn{7}{|l|}{$\mathrm{p} 53$} \\
\hline $\mathrm{LI}<50 \%$ vs. $\mathrm{LI} \geq 50 \%$ & 2.921 & $1.117-7.638$ & 0.029 & 0.960 & $0.254-3.821$ & 0.952 \\
\hline \multicolumn{7}{|l|}{ mtTFA } \\
\hline $\mathrm{LI}<32 \%$ vs. $\mathrm{LI} \geq 32 \%$ & 3.395 & $1.232-9.358$ & 0.018 & 1.224 & $0.403-3.719$ & 0.722 \\
\hline
\end{tabular}

may interrupt the ability of cancer cells to compensate for oxidative stress.

The mitochondria produce cellular energy by oxidative phosphorylation and generate reactive oxygen species (ROS) as a by-product. The mitochondrial genome exhibits higher mutation rates than the nuclear genome and is highly susceptible to damage caused by ROS [13]. Oxidative stress and resultant mtDNA mutations have been suggested to underlie the development and/or maintenance of the malignant phenotype [14]. The D-loop region of mtDNA is a hotspot for somatic mutations in human cancers [14]. A somatic D-loop mtDNA mutation was found in five $(62.5 \%)$ of eight uterine serous carcinomas [15]. Authors suggested that oxidative damage is an important mechanism in the development of serous carcinoma. The mtTFA expression of nonendometrioid carcinomas was not associated with the clinicopathologic variables and prognosis of the patients. In contrast, the mtTFA expression of endometrioid adenocarcinomas was significantly associated with the clinicopathologic variables and prognosis of the patients. A large study for nonendometrioid carcinoma is necessary to resolve this question. There is no literature concerning with the incidence of mtDNA mutation in endometrioid adenocarcinomas or correlation between mtTFA expression and mutation of mtDNA.

Using a p53 index of $\geq 50 \%$ as a cut-off between positive and negative p53 staining, immunohistochemical staining for $\mathrm{p} 53$ was reported to be a significant prognostic indicator in patients with endometrioid adenocarcinoma [11]. This result was confirmed in the present study. The aggressive clinical course and high p53 expression of nonendometrioid carcinomas were also confirmed. Yoshida et al. suggested that the physical and functional interaction of p53 with mtTFA play an important role in apoptosis [10]. An increased apoptosis was observed in mtTFA knockout animals, thus suggesting that mtTFA is involved in apoptosis [16]. The cell lines of radio-resistant oral squamous cell carcinoma strongly expressed mtTFA in comparison to those of the radio-sensitive oral squamous cell carcinoma [17]. Chemotherapy and radiation treatment of cancer are intended to induce apoptosis in tumor cells. It is interesting that correlation analysis between mtTFA and p53 expression by using the Pearson test showed significant correlation in endometrioid adenocarcinomas.

In conclusion, the positive mtTFA expression is a useful maker for the progression of the tumors and the poor prognosis of the patients in endometrioid adenocarcinomas. Immunohistochemical expression of p53 was a prognostic indicator in patients with endometrioid adenocarcinoma. The mtTFA activity of endometrioid adenocarcinomas may play a different role from that of nonendometrioid carcinomas, through initiation and/or progression of the cancerous processes.

Conflict of interest statement We declare that we have no conflict of interest. 
Open Access This article is distributed under the terms of the Creative Commons Attribution Noncommercial License which permits any noncommercial use, distribution, and reproduction in any medium, provided the original author(s) and source are credited.

\section{References}

1. Sherman ME (2000) Theories of endometrial carcinogenesis: a multidisciplinary approach. Mod Pathol 13:295-308

2. Hecht JL, Mutter GL (2006) Molecular and pathologic aspects of endometrial carcinogenesis. J Clin Oncol 24:4783-4791

3. Ekstrand MI, Falkenberg M, Rantanen A et al (2004) Mitochondrial transcription factor A regulates mtDNA copy number in mammals. Hum Mol Genet 13:935-944

4. Gaspari M, Lasson NG, Gustafsson CM (2004) The transcription machinery in mammalian mitochondria. Biochim Biophys Acta 1659:148-152

5. Kanki T, Ohgaki K, Gaspari M et al (2004) Architectural role of mitochondrial transcription factor $\mathrm{A}$ in maintenance of human mitochondrial DNA. Mol Cell Biol 24:9823-9834

6. Jeng JY, Yeh TS, Lee JW et al (2008) Maintenance of mitochondrial DNA copy number and expression are essential for preservation of mitochondrial function and cell growth. J Cell Biochem 103:347-357

7. Wang Y, Liu VWS, Xue WC et al (2005) The increase of mitochondrial DNA content in endometrial adenocarcinoma cells: a quantitative study using laser-captured microdissected tissues. Gynecol Oncol 98:104-110

8.. Cormio A, Cuerra F, Cormio G, et al. (2009) The PGC-1 $\alpha$-dependent pathway of mitochondrial biogenesis is upregulated in type I endometrial cancer. Biochem Biophys Res Commun (in press)

9. Lin PC, Lin JK, Yang SH et al (2008) Expression of $\beta$-F1-ATPase and mitochondrial transcription factor $\mathrm{A}$ and the change in mitochondrial DNA content in colorectal cancer: clinical data analysis and evidence from an in vitro study. Int J Colorectal Dis 23:1223-1232

10. Yoshida Y, Izumi H, Torigoe $\mathrm{T}$ et al (2003) p53 physically interacts with mitochondrial transcription factor A and differentially regulates binding to damaged DNA. Cancer Res 63:3729-3734

11. Alkushi A, Lim P, Coldman A et al (2004) Interpretation of p53 immunoreactivity in endometrial carcinoma: establishing a clinically relevant cut-off level. Int J Gynecol Pathol 23:129-137

12. Lee HC, Yin PH, CY LU et al (2000) Increase of mitochondria and mitochondrial DNA in response to oxidative stress in human cells. Biochem J 348:425-432

13. Yakes FM, Van Houten B (1997) Mitochondrial DNA damage is more extensive and persists longer than nuclear DNA damage in human cells following oxidative stress. Proc Natl Acad Sci USA 94:514-519

14. Modica-Napolitano JS, Kulawiec M, Singh KK (2007) Mitochondria and human cancer. Current Molecular Medicine 7:121-131

15. Pejovic T, Ladner D, Intengan M et al (2004) Somatic D-loop mitochondrial DNA mutations are frequent in uterine serous carcinoma. Eur J Cancer 40:2519-2524

16. Wang J, Silva JP, Gustafsson CM et al (2001) Increased in vivo apoptosis in cells lacking mitochondrial DNA gene expression. PNAS 98:4038-4043

17. Ueta E, Sasabe E, Yang Z et al (2008) Enhancement of apoptotic damage of squamous cell carcinoma cells by inhibition of the mitochondrial DNA repairing system. Cancer Sci 99:22302237 